\title{
Advancing with Ceramic Biocomposites for Bone Graft Implants
}

\author{
Nathan Zaidman ${ }^{1}$ and Darko Bosnakovski ${ }^{2 *}$ \\ ${ }^{I}$ Department of Medicine, University of Minnesota, Minneapolis, MN 55455, USA; ${ }^{2}$ Faculty of Medical Sciences, \\ University "Goce Delcev" Stip, Krste Misirkov bb, 2000 Stip, R. Macedonia
}

Received: November 1, 2010; Accepted: December 31, 2010; Revised: August 23, 2011

\begin{abstract}
Bone grafting has come a very long way since a Dutch surgeon used pieces of a dog's skull to repair a soldier's cranium in the $17^{\text {th }}$ Century. Current technology aims to deliver a scaffold that combines the unique osteogenic properties of ceramic biocomposite materials to make the best mimic of physiologic conditions. To do so, a scaffold must provide: i) A three-dimensional platform allowing for osteogenic cellular attachment and growth and vascular formation, ii) Structural integrity while the damaged tissue heals, and iii) Non-toxic integration, degradation or resorption into the host over an appropriate time. The combination of inorganic, ceramic materials with cells, polymers and growth factors has come very close to creating a bone graft capable of meeting each of these requirements. Recent patents describe new methods to forming an ideal osteogenic matrix for both large and small bone repair. Many new technologies have been introduced that are very potent in their ability to heal small bone wounds and induce new bone formation, such as porous calcium phosphate pastes and hydroxyapatite cements. However, there is still a lack of quality and proven materials for load bearing purposes. This is a reminder of how much there still is to improve upon and that we are still a long way from creating bone products that are identical to the natural product. Despite these shortcomings, ceramic biocomposties represent one of the most promising materials in the bone graft field and their development and improvement will surely lead to a more natural bone replacement.
\end{abstract}

Keywords: Bioactive, bioceramic, biocomposite, ceramic, orthopedic implants, stem cell, tissue engineering.

\section{INTRODUCTION}

Both the natural science and engineering communities have contributed to the rapidly advancing field of bone repair. Bone grafts require particular mechanical properties as well as an enormous degree of biocompatibility. Thanks to this collaborative effort, many new products are able to offer top degrees of both design aspects. In this short review, we will present a brief run-up to the current state of bone graft technology and analyze patents which we find especially exciting for the future of the field.

Bone grafting and bone repair have changed dramatically since a Dutch surgeon used pieces of a dog's skull to repair a soldier's cranium in the $17^{\text {th }}$ Century [1]. Autografts, the gold standard of tissue transplantation and bone repair, provide an almost perfect scaffold for bone grafting, as there is little to no chance of rejection by the host and an already developed structural and vascular network. However, autografts usually require a second surgery, meaning additional wound, prolongated recovery time, more risk of infection, as well as being severely limited in supply [1-4]. Allografts also provide an excellent material for bone grafting but have the added risks of rejection and disease transmission. To overcome the difficulties with autografts and allografts, scaffolds designed to repair and permit regeneration of bone have been produced from an array of materials each having a unique set

*Address correspondence to D.B. at University "Goce Delcev" Stip, Faculty of Medical Sciences, Krste Misirkov bb, 2000 Stip, R. Macedonia;

Tel: 389 (0)70 516649; Fax: 389 (32) 550 001;

E-mail: darko.bosnakovski@ugd.edu.mk of mechanical and biologically relevant properties. These materials include natural polymers (collagen, chitin, alginate), synthetic polymers (Polyglycolic acid (PGA), Poly (lactic-co-glycolic acid) (PLGA), Poly (lactic acid) (PLA)), metals (titanium, nitinol), and ceramics such as calcium phosphates (hydroxyapatite, tricalcium phosphate), calcium sulphates, and biological glass Fig. (1). It should be noted that there is great diversity between materials even belonging to the same product group. For instance, hydroxyapatite is rather inert whereas other ceramics are resorbed over a large range of times.

Current technology aims to deliver a scaffold that combines the unique osteogenic properties of these materials to make the best biomimic of physiologic conditions. To do so, a scaffold must provide: i) A three-dimensional platform allowing for vascular formation and osteogenic cellular attachment and growth, ii) Structural integrity while the damaged tissue heals, and iii) Non-toxic integration, degradation or resorption into the host over an appropriate time [5-7].

The first requirement can be met by using biocompatible materials that allow for cell growth (an osteoconductive material) $[1,5,8,9]$ or by the attachment of signaling molecules to the material, which themselves promote the attachment and proliferation of relevant cell populations. Adult stem cells including bone marrow mesenchymal stem cells (MSCs), MSC from adipose tissue, cord blood stem cells, as well as differentiated embryonic stem cells (ESCs), when combined with an appropriate 3D scaffold, have been shown to be capable of producing bone in vivo Fig. (1) [10-16] MSCs can provide a large amount of potential osteogenic cells for use in tissue engineering and offer great excitement 


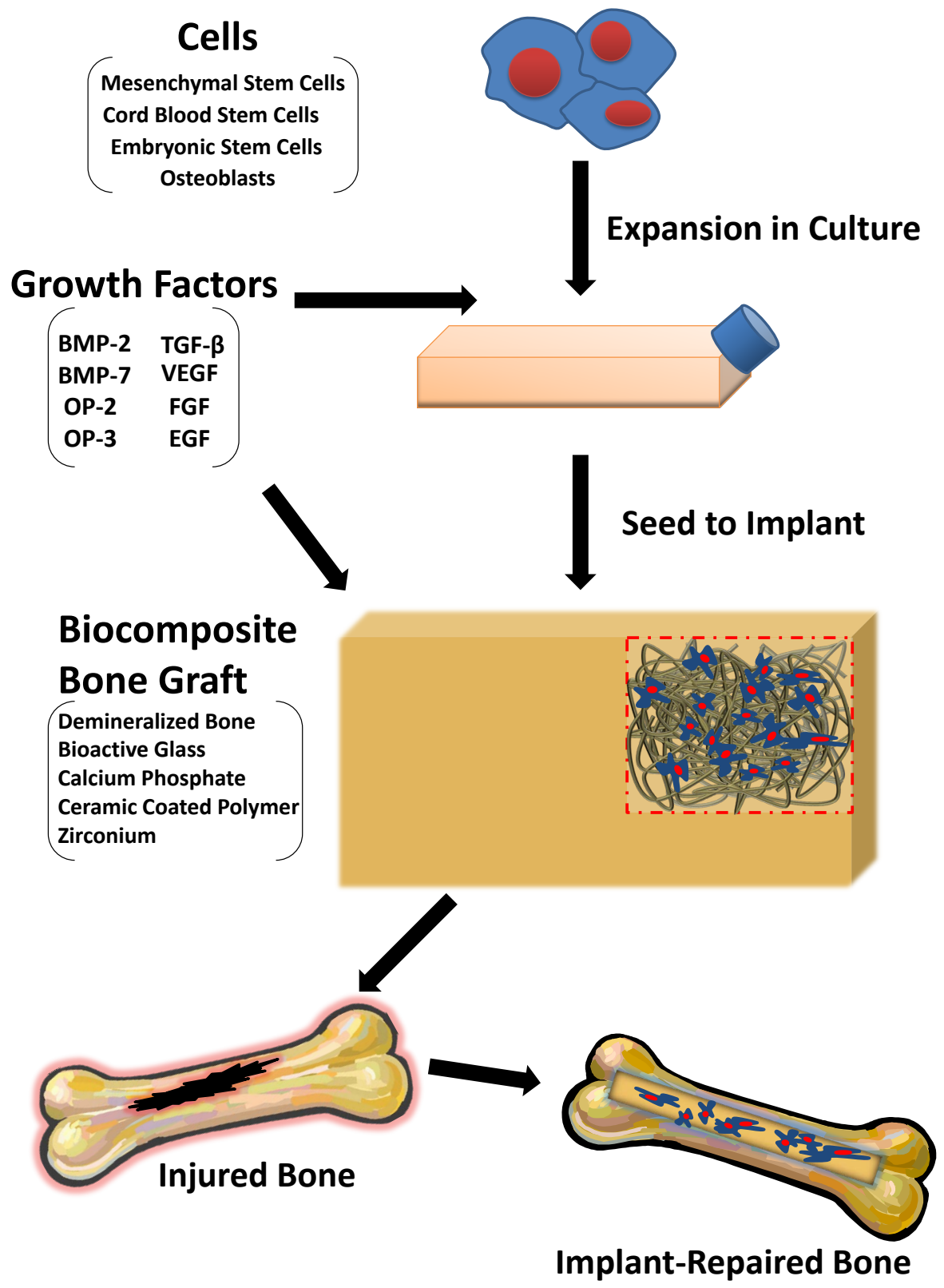

Fig. (1). Steps for creating a cell-seeded biocomposite bone implant. Scheme showing the process of preparing a biocomposite bone material for implantation using a cell-seeding technique as well as growth factors to promote osteogenic lineage. First, osteogenic cells are expanded in culture to produce sufficient cell number. The cells may be derived from adult stem cells such as bone marrow mesenchymal stem cells (MSCs), MSC from adipose, cord blood stem cells, as well as differentiated embryonic stem cells. Each of these cell types has been shown to produce bone in vivo. Next, the addition of growth factors may or may not be necessary to drive cellular differentiation, depending on the material. For example, bioactive glass is capable of producing ossified tissue without the assistance of exogenous growth factors. The cells are then seeded onto the biocomposite bone graft and allowed to expand on and into the material. The material (demineralized cadeveric bone, bioactive glass, zirconium, calcium phosphate, etc.) is to be made porous to allow new bone formation in all three-dimensions. The implant is then placed at the site of injury and, over time, completely integrates into the host tissue.

for the future of bone grafting. Signaling molecules such as bone morphogenic proteins (BMP-2, BMP-7/OP-1, OP-2, OP-3), transforming growth factor beta (TGF-beta), vascular endothelial growth factor (VEGF), fibroblast growth factor (FGF), epidermal growth factor (EGF) among many others are known osteogenic proteins that are capable of inducing a progenitor cell to form bone and/or cartilage tissue Fig. (1) $[1,4,17,18]$. Furthermore, we and others showed that extracellular matrix independent from other factors can also induce differentiation of bone marrow MSC [19-21]. The combination of cells, molecules and materials is essential to providing a scaffold with osteoconducting and osteoinducting properties.

Equally important to the proliferation of osteogenic cells is the development of a functioning vascular supply to the growing bone graft, particularly very large ones that become necrotic in their centers when wholly dependent on diffusive transport of oxygen [22]. Strategies to produce a vascular 
network include using a mix of MSCs and umbilical cordderived endothelial cells to seed a scaffold [23], targeting proximal vasculature to integrate and expand into the graft using cytokines [24], or simply implanting a vascular bundle to the injury and graft site [25]. The construction of a complete vascular system is the single most important factor in determining the success of an orthopedic implant.

A major concern of bone grafts is the ability to bear significant load while the damaged tissue heals [5]. Furthermore, a proper implant should not overtake the loads of proximal tissue as this may cause the healthy bone to weaken, called stress shielding [26]. Additional to these very specific mechanical properties, a scaffold must be porous enough to allow osteoblasts or other progenitor cells to populate the entire scaffold and osseointegrate with surrounding tissue $[5,27]$. The need for vascular networks also requires much larger pores in the bone graft material than would otherwise be needed, adding yet another challenge to scaffold design [28].

The third requirement is possibly the most difficult to provide, since bone in-growth occurs differently at all injury sites and must be finely tuned so as not to cause more harm $[2,3]$. A proper scaffold should be completely and totally replaced by regenerated tissue, that is, the patient should not depend on the mechanics of the scaffold long after implantation. Rates of degradation of materials vary greatly, as well as the location at which they begin to erode (surface, bulk, both). Materials that are biodegradable, bioresorbable, bioerodible and bioabsorbable must also retain the properties to meet the first two requirements [2, 5, 18, 27, 29]. Therefore, the search for the perfect bone tissue scaffold will require a great deal of arrangement to generate a whole greater than the sum of the parts.

These materials, which combine structural and biologic properties from different materials, are known as biocomposites. Containing cells, signaling molecules, or a combination of the two within a structural matrix, biocomposites are biologically active materials with great promise for tissue engineering. Here, we give a brief review of the most current technology pertaining to the use of ceramic biocomposites in bone grafts.

\section{RECENTLY ISSUED PATENTS ON CERAMIC BONE GRAFT MATERIALS}

\section{Natural Ceramics, Endochondral Ossification and Bioac- tive Glass}

The most obvious means of recreating bone is to simply replace the tissue with already formed bone. A recent patent published May 2010 by inventor Shi titled Allografts Combined with Tissue Derived Stem Cells for Bone Healing, describes a method of using donor bone for use as a scaffold for bone formation from adipose-derived mesenchymal stem cells (Table 1) [30]. The bone substrate, which is to be retrieved from a cadaveric donor, may be seeded with adipose tissue either from the same cadaveric donor or the implant recipient. The patent also describes a demineralization process to remove blood, lipids and other cellular products that may initiate an immune response, leaving just a matrix for the MSCs to adhere to. The bone may then be formed into any number of necessary shapes, such as strips and dowels. Once the bone is properly prepared, the author recommends in vitro culturing of the cells until they have adhered to the bone substrate before implantation. This process takes advantage of a scaffold produced in nature, requiring no outside engineering, as well as the advantages of using patient MSCs for osteogenesis. The porosity of the bone substrate is not well manipulated however, and may not be well suited for small repair operations. This method is probably best served when relatively large implants are required or when a matrix is called for with in vitro study.

Another method that uses an equally natural approach is presented by Jukes et al., published in PNAS May 2008 [13]. Following the model of endochondral ossification, the authors use ESCs to first produce a cartilage layer atop a ceramic scaffold before implanting the product. Since ESCs having up-regulated osteogenic genes do not form bone when implanted, as opposed to osteogenic MSCs, it is necessary to follow the course nature uses by differentiating the ESCs into chondrocytes. The ESCs are therefore differentiated in vitro towards the chondrogenic lineage and seeded onto the ceramic material. Upon implantation, these cartilage tissue-engineered constructs do indeed form bone, in stark contrast to constructs seeded with ESCs differentiated towards an osteogenic lineage. While this method produces bone having a much more natural derivation, the issues surrounding ESCs implantation, such as tissue rejection, persist, once again pointing towards the benefit of MSCs use.

Synthetically produced materials amass the vast majority of biocomposite materials. One such ceramic that offers a great deal of excitement is bioactive glass. Discovered in 1969 and first used in clinical settings in 1985, bioactive glasses have a very unique property that promotes osteoblasts maturation into ostocytes [31]. The mechanism believed to be responsible for this is the constant leeching of ions from the dissolution products of the glass that results in the up-regulation of certain genes that are implicated in osteogenesis (insulin like growth factor II (IGF-II), CD44, cMyc, activator protein $1(\mathrm{AP}-1))[4,31]$. In a patent published in January, 2002 by Hench et al. titled Use of Bioactive Glass Compositions to Stimulate Osteoblast Production the authors illustrate a more patch like method of applying the bioactive ceramic (Table 1). The patent describes the use of bioactive glass powder in an aqueous solution combined with osteoblasts for the formation of bone tissue. As published, the bioactive glass is to contain a majority silicon dioxide by weight, as well as sodium oxide, calcium oxide and phosphorous oxide and is to be produced as a particulate rather than a "fused matrix of particles or a mesh or fabric of glass fibers." This allows for extreme customization in the hands of the surgeon or researcher. However, the product is only to be used as an initiator to bone formation, not as a structural implant due to the lack of strong mechanical properties. Therefore, the use of bioactive glass in this manner is best used for small repairs, tissue culture, or combined with some other scaffold material having much better mechanical strength. 
Table 1. Natural Ceramic and Bioactive Glass Patents.

\begin{tabular}{|l|l|l|}
\hline \multicolumn{1}{|c|}{ Patent \# (Year) } & \multicolumn{1}{|c|}{ Inventor(s) } & \multicolumn{1}{c|}{ Description } \\
\hline \hline US20100124776 (2010) & Shi Y & $\begin{array}{l}\text { A method for seeding a bone substrate with mesenchymal stem cells (MSCs). The inventor } \\
\text { describes the digestion of a whole tissue, seeding the cell mixture on a bone substrate, cultur- } \\
\text { ing on the bone substrate and rinsing to remove unwanted cells. }\end{array}$ \\
\hline US20040009598 (2004) & $\begin{array}{l}\text { Hench LL } \\
\text { Polak JM } \\
\text { Buttery LDK } \\
\text { Xynos ID } \\
\text { Maroothynaden J }\end{array}$ & Bioactive glass powder combined with osteoblasts in an aqueous solution used as a cement. \\
\hline
\end{tabular}

\section{Ceramic-Polymer Composite Materials}

A good example of a scaffold that is coated with a bioactive material is presented in a patent published March 2009 by Johnson and Tavakley (Table 2) [18]. Titled Composite Scaffold Structure, the authors describe a scaffold design having a polymeric core that is discretely coated with calcium phosphate, resulting in a porous scaffold with a high amount of surface area. The use of a polymeric core ensures the flexibility of the scaffold and allows for a compressive strength similar to cartilage, for which the invention was designed. The authors outline a variety of materials that may be used as the core structure including Poly(lactic acid) (PLA), Polyglycolic acid (PGA), Poly(lactic-co-glycolic acid) (PLGA), polycaprolactone, and polyanhydrides as well as natural materials like collagen, albumin, fibrin and alginate, all of which are bioremovable, that is, they will be completely removed through degredation from the final scaffold. The surface coating of calcium phosphate, which facilitates the attachment and growth of cells on the scaffold, is said to be discrete so as to allow the polymeric core to maintain its lower elastic modulus. An entire surface coat would render the structure much more brittle, such that it could crack or break under normal body stresses.

This idea of using mechanical properties of one material along with the bioactive properties of another is very similar to the actual structure of bone. Bone itself contains a precise mixture of collagen fibers weaving through a harder hydroxyapatite (HA) phase. This combination allows bone to posses both bioactive and mechanical properties. Biomimics containing this very make up have been shown to support the growth and proliferation of MSCs both in vitro and in vivo [6]. In his 2007, manuscript published in Biomaterials, Dr. Huanan Wang described a method of designing a porous hydroxyapatite-polyamide structure having very similar mechanical strengths as bone, including load-bearing bone. His groups' scaffolds were able to produce bone with or without seeding of MSCs when implanted into mice.

Two patents published in 2007 and 2008 by inventors Erbe and Clineff, respectively, present another embodiment of a composite containing a polymeric phase (Table 2) [32, 33]. Both patents describe a resorbable graft having improved flexibility and compressive strength. The bone graft material is comprised of an oxidation-reduction reaction product of "one metal cation, one oxidizing agent, and one oxidizable precursor anion," such as calcium phosphate, combined with a biocompatible, resorbable polymer, such as collagen or another structural protein. Another embodiment of the material contains a bioactive glass, as well, to promote osteoblast growth. The 70/30 volumetric ratio of reaction product to polymer creates a unique material having macro, meso and micropores due to the reaction product as well as beneficial handling and flexibility from the polymer. The porosity leads to outstanding imbibition and protein adsorption, while the flexibility of the material allows a great deal of customization in the hands of the surgeon.

A patent published in 2004 from inventor Wang introduces another variation on the combination of polymer and ceramic calcium phosphate (Table 2) [34]. Here, the polymer is used simply to create pores in the ceramic medium. One embodiment of the invention calls for a composite mixture of calcium phosphate cement, an aqueous medium and a biodegradable polymer as a porogen. The porogen can be finely tuned to degrade at the exact rate of osteoblast reseeding of the calcium phosphate matrix and the inventor even describes a porogen filled with osteoinductive growth factors. This material is once again best suited for small repair jobs or coupled with another bone graft system to increase the osteoconductiveness of the implant.

More recently, a patent published in February 2011 from inventor Yayon describes yet another bone graft material comprised of an inorganic mineral phase as well as a bioactive polymer (Table 2) [35]. The invention goes one step further in the formation of bone to include an anti-resorptive agent, such as bisphosphonates. These agents inhibit the recruitment or activity of osteoclasts, the bone resorbing cells responsible for the subtractive side of bone remodeling. Rather than simply promoting osteoblast activity, the inventors have taken an approach that aims to protect the newly formed, delicate bone from the natural process all bone experiences in vivo. The composite material is formed by coprecipitation of calcium, phosphate, and carbonate ions, one amino acid molecule either as a monomer or polymer, a bioactive agent such as heparin, hyaluronic acid, starch, collagen, chitin or the like, and the anti-resorptive agent. The final product is deemed a synthetic apatite having poor crystallinity that highly mimics the structure of natural bone. The inventors specifically avoid hydroxyapatite due to its high de- 
Table 2. Ceramic-Polymer Composite Material Patents.

\begin{tabular}{|c|c|c|}
\hline US20090062821 (2009) & $\begin{array}{l}\text { Johnson JR } \\
\text { Tavakley A }\end{array}$ & Polymer scaffold having a discrete, non-continuous coating of calcium phosphate on the surface. \\
\hline $\begin{array}{l}\text { US7189263 } \\
(\mathbf{2 0 0 7 )}\end{array}$ & $\begin{array}{l}\text { Erbe EM } \\
\text { Clineff TD } \\
\text { Bagga CS } \\
\text { Nagvajara G M } \\
\text { Koblish A }\end{array}$ & $\begin{array}{l}\text { Bioactive ceramic phase such as calcium phosphate, mixed with a biocompatible, resorbable } \\
\text { polymer, such as collagen. The porous material is greatly flexible and displays high adsorptive } \\
\text { properties. }\end{array}$ \\
\hline $\begin{array}{l}\text { US20080187571 } \\
(\mathbf{2 0 0 8})\end{array}$ & $\begin{array}{l}\text { Clineff TD } \\
\text { Koblish A } \\
\text { Bagga CS } \\
\text { Erbe EM } \\
\text { Nagvajara G M } \\
\text { Darmoc MM }\end{array}$ & Same as above, except with the addition of bioactive glass. \\
\hline $\begin{array}{l}\text { US20040137032 } \\
\text { (2004) }\end{array}$ & Wang FW & $\begin{array}{l}\text { Calcium phosphate mixed with a biodegradable polymer acting as a porogen. Best suited as a } \\
\text { cement material. }\end{array}$ \\
\hline $\begin{array}{l}\text { US7887831 } \\
\text { (2011) }\end{array}$ & Yayon A & $\begin{array}{l}\text { Composite of synthetic apatite, a bioactive polymer and an anti-resorptive agent, limiting osteo- } \\
\text { clast activity. }\end{array}$ \\
\hline
\end{tabular}

gree of crystallinity, claiming less organized structures improve solubility in vivo and thus allow the material to be used as a powder or paste.

\section{Osteoinductive Calcium-Phosphate Grafts without Poly- mers}

Scaffolds produced purely from calcium phosphate ceramics, without a polymeric phase, are themselves capable of supporting MSCs and initiating bone formation [36]. The clear advantage of producing a biocomposite containing both polymeric and ceramic materials is an elastic modulus much lower than the pure ceramic. However, if a ceramic material can be made to be as dynamic as a polymer, one could remove the polymeric material all together, as it is not the bioactive phase. A recent patent published in June 2010, describes such an invention [2]. Authors Day and Erbe reveal a dynamic, nonrigid, nanofiber scaffold produced from bioactive materials to be combined with progenitor cells (Table 3 ). These materials may include but are not limited to calcium phosphate, hydroxyapatite, and bioactive glass. Used alone or in combination with others, the material is spun or blown into an interwoven network of 1 to 6 micron diameter fibers, depending on desired porosity. Since the scaffold is not fused to itself the individual fibers are free to deform and shift due to environmental changes. The scaffold is designed to be highly dynamic before and during osseointegration. This is a very important trait as the injury site will change drastically as it heals, possibly over the course of many months. The invention may also be coupled with previous devices, such as the selectively-expandable bone scaffold described in a November 2006 patent published by Edidin, which uses an actuator to maximize the dynamic nature of the scaffold Table 3 [37]. The flexibility of the authors' invention, as well as its bioactivity, represents an exciting leap with ceramic scaffolds.

Another patent which focuses solely on calcium phosphate was published May 2011 by inventor Yuan (Table 3) [38]. The patent introduces a very porous calcium phosphate material made using grains of the raw material ranging from 0.1 to 1.5 microns in diameter. When sintered with a porogen, the final material contains mainly micropores ranging from 0.1 to 1.5 microns in diameter and having a surface area percent micropores of $40 \%$. The invention stems from the idea that small grain sizes, higher surface area percentages of micropores and higher protein adsorption all increase the osteoconductiveness of the material. Here the author aims to simply produce a material that can act much quicker than less porous calcium phosphate graft materials. Due to the sand-like nature of the material, the inventor envisions the product being injected into wound sites and rapidly enhancing new bone formation.

\section{Load Bearing Zirconia Grafts}

Although dynamic, shape-changing ceramic scaffolds give highly customizable results, they may not always be best suited for major bone grafts, requiring intense loadbearing properties. One material that is able to withstand these stresses is zirconia (zirconium dioxide). Zirconia has been extensively studied for orthopedic applications due to its high elastic modulus [39]. However, the material itself 
Table 3. Osteoinductive Calcium-Phosphate Grafts Without Polymer Patents.

\begin{tabular}{|l|l|l|}
\hline \multicolumn{1}{|c|}{ Patent \# (Year) } & \multicolumn{1}{|c|}{ Inventor(s) } & \multicolumn{1}{c|}{ Description } \\
\hline \hline US20100136086 (2010) & $\begin{array}{l}\text { Day TE } \\
\text { Erbe EM }\end{array}$ & $\begin{array}{l}\text { Dynamic, non-rigid nanofiber scaffold made from bioactive ceramic, such as calcium } \\
\text { phospate, bioactive glass, or hydroxyapatite. }\end{array}$ \\
\hline US20060264945 (2006) & $\begin{array}{l}\text { Edidin AA } \\
\text { Ferdinand AE } \\
\text { Ico CA } \\
\text { Markworth AD }\end{array}$ & $\begin{array}{l}\text { Dynamic ceramic scaffold surrounding a mechanical actuator. The actuator allows the } \\
\text { scaffold to expand into the wound and shrink as it heals. }\end{array}$ \\
\hline US7942934 (2011) & $\begin{array}{l}\text { Yuan H } \\
\text { Bruijn JDD }\end{array}$ & $\begin{array}{l}\text { Porous calcium phosphate scaffold made from granules of calcium phosphate. The } \\
\text { material's flow properties resemble those of sand. }\end{array}$ \\
\hline
\end{tabular}

has a very inert surface, as most ceramics do, which limits interaction with surrounding tissue. More specifically, the surface does not form biochemical bonds, thus leading to in situ slippage [26, 40]. Therefore, to fully take advantage of the mechanical strength of zirconia it must first be coated with a biologically active material. One interesting approach was published in January 2010 in the journal Biomaterials by Zhou et al. [41]. The authors present a method of coating zirconia with a sheet of cultured MSCs. The process requires a monolayer of cells to be rolled up such that it completely encapsulates a zirconia implant. The wrapped implants were shown to contribute heavily to in vivo bone formation and osseointegration when implanted subcutaneously in mice following histological analysis. This approach represents a novel technique in priming ceramic surfaces for implantation, but is still far from perfect.

In a patent published in May 2007 by inventor Sugaya, the author describes a porous, ceria stabilized zirconia for use as an osteoinductive scaffold (Table 4) [17]. The use of cerium oxide to stabilize zirconia produces an oxygen rich layer at the surface of the zirconia about 100 to $300 \mathrm{~nm}$ thick. This produces a highly biocompatible product, suitable for MSC or osteoblast seeding, along with high strength and fracture toughness. Another advantage of the dynamic and customizable material is that it may be shaped into any number of appropriate forms. The relatively easy preparation of the ceria-stabilized zirconia (plasma spray) allows the material to be attractive for both research and tissue repair applications. The clear drawback of zirconia is that it lacks any bioremovable characteristics, resulting in a permanent scaffold structure. However, due to the porosity of the implant, osseointegration may completely annul any complications due to implant-slippage overtime.

Another recent invention that takes advantage of the mechanical strength of zirconia as well as the bioactive properties of calcium phosphate is presented in a patent published in May 2010 by Zhang et al. (Table 4) [26]. The inventors describe a material having a strong zirconia core and a bioactive, but mechanically weaker, glass surface connected through a graded glass-zirconia layer. The material is fabricated in such a way by using a glass-ceramic infiltration technique. This method produces a material having an increasing elastic modulus from the surface to the interior, which may then be spun or molded into any number of porous scaffold structures. An advantage of this type of graded material is that it provides the scaffold with excellent resistance to contact damage. In fact, when the authors subject a sample of their graded material to loading tests they find that it is able to resist over three times the load it takes to crack a sample of monolithic zirconia. The production of a material that has a low modulus, bioactive surface while still having high flexural strength is quite advantageous and has numerous applications in the field.

\section{CURRENT \& FUTURE DEVELOPMENTS}

Biocomposites represent the future of material science in the medical setting, especially in orthopedic implantation. Porous ceramic scaffolds have already been noted as the most suitable material for reproducing the structural integrity of ossified tissues. When combined with the bioactive attributes of calcium phosphate, hydroxyapatite, bioactive glass, or other similar ceramics, the composite material is able to support progenitor cells and mimic the natural characteristics of bone. Whether it is applying new technologies to old problems, as Shi was able to accomplish with donor bone, or conquering the boundary between polymers and ceramics, like Day and Erbe demonstrated with their hydroxyapatite "cotton ball", biocomposites of this nature encompass a broad spectrum of regenerative scaffolds and bone grafting needs. However, though current technology is able to produce accurate biomimics of natural bone, there is still much to be gained; none of these presented materials is quite able to meet the standard of an autograft implant and all would require a certain degree of invasiveness to deploy. Furthermore, there is still a large gap to be covered with vascularized implants. That said, overcoming boundaries such as immune responses, osseointegration and material resorption are all feats to be proud of and to build off of for future generations of bone grafts.

\section{ACKNOWLEDGEMENTS}

Darko Bosnakovski laboratory was partially supported by AFM (2011-205/ 15380) and "FSH Society Research Fellowship grant FSHS-82010-01”. 
Table 4. Load Bearing Zirconia Graft Patents.

\begin{tabular}{|l|l|l|}
\hline \multicolumn{1}{|c|}{ Patent \# (Year) } & \multicolumn{1}{|c|}{ Inventor(s) } & \multicolumn{1}{c|}{ Description } \\
\hline \hline US20070123996 (2007) & $\begin{array}{l}\text { Sugaya K, Merchant S } \\
\text { Seal S, Georgieva P } \\
\text { Vrotsos M }\end{array}$ & $\begin{array}{l}\text { Ceria stabilized zirconia scaffold. Mechanically strong and bioactive, due to an oxygen rich } \\
\text { layer at the zirconia surface. }\end{array}$ \\
\hline US20090118114 (2009) & $\begin{array}{l}\text { Zhang Y, Regeros R } \\
\text { Kim JW }\end{array}$ & $\begin{array}{l}\text { Graded glass-zirconia graft material, having a strong zirconia core and a bioactive surface } \\
\text { containing bioglass. }\end{array}$ \\
\hline
\end{tabular}

\section{CONFLICT OF INTEREST}

The authors do not know of any conflicts of interest.

\section{REFERENCES}

[1] Jahangir A, Nunley RM, Mehta S, SharanA, and the Washington Health Policy Fellows. Bone-graft substitutes in orthopaedic surgery. AAOS Now 2; 2008.

[2] Day, T.E., Erbe, E.M. Dynamic bioactive nanofiber scaffolding. US20100136086 (2010).

[3] Laurencin C, Khan Y, El-Amin SF. Bone graft substitutes. Expert Rev Med Devices 2006; 3: 49-57.

[4] Hench, L.L., Polak, J .M., Buttery, L.D.K., Xynos, I.D., Maroothynaden, J. Use of bioactive glass compositions to stimulate osteoblast production. US20040009598 (2004).

[5] Hutmacher DW. Scaffolds in tissue engineering bone and cartilage. Biomaterials 2000; 21: 2529-43.

[6] Wang $\mathrm{H}, \mathrm{Li} \mathrm{Y}, \mathrm{Zuo} \mathrm{Y}, \mathrm{Li} \mathrm{J}$, Ma S. Biocompatibility and osteogenesis of biomimetic nano-hydroxyapatite/polyamide composite scaffolds for bone tissue engineering. Biomaterials 2007; 28: 3338-48.

[7] Puleo DA, Nanci A. Understanding and controlling the boneimplant interface. Biomaterials 1999; 20: 2311-21.

[8] Langstaff S, Sayer M, Smith TJ, Pugh SM, Hesp SA. Resorbable bioceramics based on stabilized calcium phosphates. Part I: Rational design, sample preparation and material characterization. Biomaterials 1999; 20: 1727-41.

[9] Langstaff S, Sayer M, Smith TJ, Pugh SM. Resorbable bioceramics based on stabilized calcium phosphates. Part II: Evaluation of biological response. Biomaterials 2001; 22: 135-50.

[10] Friedenstein AJ, Piatetzky S, II, Petrakova KV. Osteogenesis in transplants of bone marrow cells. J Embryol Exp Morphol 1966; 16: 381-90.

[11] Chen F, Zhou Y, Barnabas ST, Woodruff MA, Hutmacher DW. Engineering tubular bone constructs. J Biomech 2007; 40 Suppl 1: S73-9.

[12] Ohnishi H, Oda Y, Ohgushi H. Stem cell technology using bioceramics: Hard tissue regeneration towards clinical application. Sci Technol Adv Mater 2010; 11:1: 014110.

[13] Jukes JM, Both SK, Leusink A, Sterk LM, van Blitterswijk CA. Endochondral bone tissue engineering using embryonic stem cells. Proc Natl Acad Sci USA 2008; 105: 6840-5.

[14] Bianco P, Robey PG. Stem cells in tissue engineering. Nature 2001; 414: 118-21.

[15] Lee OK, Kuo TK, Chen WM, Lee KD, Hsieh SL. Isolation of multipotent mesenchymal stem cells from umbilical cord blood. Blood 2004; 103: 1669-75.

[16] Goodwin HS, Bicknese AR, Chien SN, Bogucki BD, Oliver DA. Multilineage differentiation activity by cells isolated from umbilical cord blood: Expression of bone, fat, and neural markers. Biol Blood Marrow Transpl 2001; 7: 581-8.

[17] Sugaya, K., Merchant, S., Seal, S., Georgieva, P., Vrotsos, M. Stem cell comprising tissue substitutes. US20070123996 (2007).

[18] Johnson, J.R.S., Tavakley, A. Composite scaffold structure. US20090062821 (2009).
[19] Bosnakovski D, Mizuno M, Kim G, Takagi S, Okumura M. Chondrogenic differentiation of bovine bone marrow mesenchymal stem cells (MSCs) in different hydrogels: Influence of collagen type II extracellular matrix on MSC chondrogenesis. Biotechnol Bioeng 2006; 93: 1152-63.

[20] Mizuno M, Fujisawa R, Kuboki Y. Type I collagen-induced osteoblastic differentiation of bone-marrow cells mediated by collagen-alpha2beta1 integrin interaction. J Cell Physiol 2000; 184: 207-13.

[21] Batorsky A, Liao J, Lund AW, Plopper GE, Stegemann JP. Encapsulation of adult human mesenchymal stem cells within collagen-agarose microenvironments. Biotechnol Bioeng 2005; 92: 492-500.

[22] Folkman J, Hochberg M. Self-regulation of growth in three dimensions. J Exp Med 1973; 138: 745-53.

[23] Tsigkou O, Pomerantseva I, Spencer JA, Redondo PA, Hart AR Engineered vascularized bone grafts. Proc Natl Acad Sci USA 2010; 107: 3311-6.

[24] Zhou M, Peng X, Mao C, Xu F, Hu M. Primate mandibular reconstruction with prefabricated, vascularized tissue-engineered bone flaps and recombinant human bone morphogenetic protein-2 implanted in situ. Biomaterials 2010; 31: 4935-43.

[25] Zhao M, Zhou J, Li X, Fang T, Dai W. Repair of bone defect with vascularized tissue engineered bone graft seeded with mesenchymal stem cells in rabbits. Microsurgery 2011; 31: 130-7.

[26] Zhang, Y.C., Regeros, R., Kim, J.-W. Bioactive graded zirconiabased structures. US20090118114 (2009).

[27] Habraken WJ, Wolke JG, Jansen JA. Ceramic composites as matrices and scaffolds for drug delivery in tissue engineering. Adv Drug Deliv Rev 2007; 59: 234-48.

[28] Jones JR, Lin S, Yue S, Lee PD, Hanna JV. Bioactive glass scaffolds for bone regeneration and their hierarchical characterisation. Proc Inst Mech Eng H 2010; 224: 1373-87.

[29] Schmid, S.R.L., Niebur, G.L., Sumner, D.R. Bone and tissue scaffolding for delivery of therapeutic agents. US7674477 (2010).

[30] Shi, Y.L. Allografts combined with tissue derived stem cells for bone healing. US20100124776 (2010).

[31] Hench LL. The story of bioglass. J Mater Sci Mater Med 2006; 17 : 967-78.

[32] Erbe, E.M., Clineff, T.D., Bagga, C.S., Nagvajara, G.M., Koblish, A. Biocompatible bone graft material. US7189263 (2007).

[33] Clineff, T.D., Bagga, C.S., Erbe, E.M., Nagvajara, G.M., Darmoc, M. Bioactive bone graft substitute. US20080187571 (2008).

[34] Wang, F. Combinations of calcium phosphates, bone growth factors, and pore-forming additives as osteoconductive composite bone grafts. US20040137032 (2004).

[35] Yayon, A. Bone enhancing composite. US7887831 (2011).

[36] Matsushima A, Kotobuki N, Tadokoro M, Kawate K, Yajima H. In vivo osteogenic capability of human mesenchymal cells cultured on hydroxyapatite and on beta-tricalcium phosphate. Artif Organs 2009; 33: 474-81.

[37] Edidin, A.A.S., Ferdinand, A.E., Ico, C.A., Markworth, A.D. Selectively-expandable bone scaffold. US20060264945 (2006).

[38] Yuan, H., Bruijn, J.D.D. Osteoinductive calcium phosphates. US7942934 (2011).

[39] Kim HW, Kim HE, Knowles JC. Hard-tissue-engineered zirconia porous scaffolds with hydroxyapatite sol-gel and slurry coatings. J Biomed Mater Res B Appl Biomater 2004; 70: 270-7. 
[40] Clem WC, Chowdhury S, Catledge SA, Weimer JJ, Shaikh FM. Mesenchymal stem cell interaction with ultra-smooth nanostructured diamond for wear-resistant orthopaedic implants. Biomaterials 2008; 29: 3461-8.
[41] Zhou W, Han C, Song Y, Yan X, Li D. The performance of bone marrow mesenchymal stem cell--implant complexes prepared by cell sheet engineering techniques. Biomaterials 2010; 31: 3212-21. 